\title{
Editorial
}

\section{Improving nutrition in Finland}

Finland is a Northern European (Nordic) country with an economy traditionally based on agriculture, particularly milk production, and forestry. After the war, the country experienced rapid industrialisation. The interplay between a growing economy and traditional habits resulted in increased consumption of fatty dairy products, with unfavourable effects on lipid metabolism and risk of CVD. High serum cholesterol levels were found to be characteristic for lumberjacks in a health survey performed in the 1950s in Eastern Finland ${ }^{(1)}$. Subsequently, two cohorts of Finnish men were included in a prospective cross-cultural study on CHD in seven countries ${ }^{(2)}$. Men in the Eastern cohort showed the highest intake of SFA, the highest mean serum cholesterol levels and the highest mortality in CHD among the fifteen cohorts of the study. Men in the Southwestern cohort showed lower risk levels that were comparable to the levels in the Netherlands and the Unites States. At the same time middle-aged Finnish men were found to have the highest CHD mortality in the world according to the WHO statistics. Awareness of the association between diet and the risk of CHD has prompted the development of surveys and the monitoring of risk factors and nutrition in the Finnish population.

In this special issue, the subsequent development of nutrition in Finland over the past 30 years is reviewed, mainly on the basis of the activities of the National Public Health Institute, renamed after a reorganisation in 2009, the National Institute for Health and Welfare. The diet of population samples from different areas of the country was surveyed in 1967-1973, and, starting in 1982, the food habits of subsamples from the FINRISK risk factor surveys have been studied at 5-year intervals. The model, originally tested in the North Karelia project in two Eastern provinces, was expanded first to cover an area in the Southwest and subsequently the capital area in the South, and the districts of Oulu and Lapland in the North ${ }^{(3)}$. The most recent FINDIET 2007 survey indicated a definite improvement in the dietary habits of adult Finns, particularly in the fatty acid composition and in salt intake ${ }^{(4)}$. Mean serum cholesterol levels have declined by $20 \%$ during the past 25 years, and changes in dietary fat composition explain $60-65 \%$ of the decline ${ }^{(5)}$. A decline in the initially high CHD mortality began in the late 1960s and has continued ever since. Between 1982 and 1997 the age-adjusted mortality declined by 63\%, and $37 \%$ of this decline was estimated to result from serum cholesterol lowering ${ }^{(6)}$.

Several factors have been involved in the positive development observed in Finland. Availability of reduced-fat dairy and meat products has been facilitated by the product developments of the food industry. Dietary fat modification has been promoted by domestic rapeseed crops and the production of rapeseed oil, which is now the principal vegetable oil and the main ingredient of soft fat spreads used in Finland. The increased availability of imported fruit and vegetables has reduced the seasonal variation of intakes and explains the 3- to 4-fold increase in average consumption. Dietary recommendations for the population were introduced in 1981 and have been revised regularly, during recent years in collaboration with the other Nordic countries $^{(7)}$. One of the major objectives of the recommendations is to act as guidelines for planning of diets in mass catering. In Finland, findings reflecting the influence of the recommendations include healthier food choices by people who use the catering services of worksite canteens and children who consume school meals ${ }^{(8)}$ and the more balanced diet of pre-school children attending day care outside home compared with those cared for at home ${ }^{(9)}$.

Despite the decrease in classical cardiovascular risk factors and in cardiovascular mortality, obesity and the incidence of type 2 diabetes have continued to increase in Finland. More than $10 \%$ of the adult Finnish population are estimated to have type 2 diabetes, about one-half of them unaware that they have the disease. The possibilities of preventing the manifestation of type 2 diabetes in atrisk individuals were highlighted in the Finnish Diabetes Prevention Study, co-ordinated by the National Public Health Institute and conducted in the five university hospital districts of Finland. The multi-factorial programme including modest weight reduction, reduction in intakes of total fat and SFA, increased intake of fibre and regular $(>30 \mathrm{~min} / \mathrm{d})$ physical activity reduced the incidence of diabetes by $58 \%$ and prevented diabetes in all those subjects who were able to fulfil four or five of the five goals of the intervention programme ${ }^{(10)}$. The lessons learned from the present study are being applied in projects on detection and prevention of diabetes in several hospital districts across the country ${ }^{(10)}$.

The Finnish government has been slow in establishing guidelines on nutrition and incorporating them in policy, but in June 2008 the current cabinet presented a resolution on guidelines for health-enhancing physical activity and nutrition that was accepted as part of a policy programme for health promotion ${ }^{(11)}$. The resolution suggests that promotion of health-enhancing physical activity and healthy diets should take place by influencing culture, living environments, life circumstances, food products 
and health promotion structures, ensuring that all population groups have sufficient information and skills to enable them to follow a health-enhancing lifestyle.

At an international level, the diet and health situation in Finland became more widely known through the abovementioned Seven Countries Study. The subsequent North Karelia project became one of the most successful intervention programmes worldwide. It has inspired other public health initiatives, one such example being the local Norsjö project in Northern Sweden ${ }^{(12)}$. The introduction of regular and comprehensive monitoring of diet and health indicators, e.g. the FINDIET and FINRISK surveys, has yielded results that have been useful for a number of purposes, for instance monitoring, policymaking and research. In addition to their primary use at the national level, the programmes and their results have also been important at an international level, showing the utility and impact of comprehensive public health investments. The high incidence of CHD initiated research projects, contributing to improved knowledge of the role of dietary fat. The Finnish Diabetes Prevention Study is another outstanding research project, and is highlighted as one of the major advances in nutrition science during the past three decades ${ }^{(13)}$.

Trends in food consumption in Finland during the past three to four decades have been broadly similar to other Nordic countries. However, changes in, for instance, dairy, fruit and vegetable consumption started somewhat later and were more pronounced and rapid compared to other Scandinavian countries ${ }^{(14)}$. The initially high intake of saturated fat from full-fat dairy products has decreased and is now lower than in many other European countries, e.g Sweden and Denmark. Efforts to decrease sodium intake (salt) have been successful, e.g. through mandatory labelling and product development ${ }^{(15,16)}$. Taken together, these achievements serve as examples of successful public health investments.

The present supplement summarises the results from previous and on-going research projects, monitoring activities and public health initiatives in the area of nutrition in Finland. It shows the importance of clinical and applied research as a basis for public health action, together with appropriate monitoring programmes. This has contributed to the decline in CHD mortality. However, increasing trends in overweight and obesity, as well as type 2 diabetes, pose additional challenges for policy initiatives related to nutrition and physical activity.

Antti Aro

Wulf Becker

Scientific Editors

\section{References}

1. Keys A, Karvonen MJ \& Fidanza F (1958) Serum cholesterol studies in Finland. Lancet 2, 175-178.

2. Keys A, Menotti A, Karvonen MJ et al. (1986) The diet and 15 -year death rate in the seven countries study. Am J Epidemiol 124, 903-915.

3. Männistö S, Laatikainen T, Helakorpi S et al. (2010) Monitoring diet and diet-related chronic disease risk factors in Finland. Public Health Nutr (in this issue).

4. Pietinen P, Paturi M, Reinivuo H et al. (2010) FINDIET 2007 survey. Public Health Nutr (in this issue).

5. Valsta LM, Tapanainen H, Laatikainen T et al. (2010) Explaining the 25-year decline of serum cholesterol by dietary changes and use of lipid lowering medication in Finland. Publ Health Nutr (in this issue).

6. Laatikainen T, Critchley J, Vartiainen E et al. (2006) Explaining the decline in coronary heart disease mortality in Finland between 1982 and 1997. Am J Epidemiol 162, 764-773.

7. Nordic Council of Ministers (2005) Nordic Nutrition Recommendations: Integrating Nutrition and Physical Activity, 4th ed., Nord 2004:13. Arhus: Scanprint as.

8. Raulio S, Roos E \& Prättälä R (2010) School and workplace meals promote healthy food habits. Public Health Nutr (in this issue).

9. Lehtisalo J, Erkkola M, Tapanainen H et al. (2010) Food consumption and nutrient intake in day care and at home in 3-year-old Finnish children. Public Health Nutr (in this issue).

10. Lindström J, Absetz P, Hemiö K et al. (2010) Reducing the risk of type 2 diabetes with nutrition and physical activity efficacy and implementation of lifestyle interventions in Finland. Public Health Nutr (in this issue).

11. Pietinen P, Männistö S, Valsta L et al. (2010) Nutrition policy in Finland. Public Health Nutr (in this issue).

12. Weinehall L, Hellsten G, Boman K et al. (2001) Can a sustainable community intervention reduce the health gap? - 10-year evaluation of a Swedish community intervention program for the prevention of cardiovascular disease. Scand J Public Health 56, Suppl., 59-68.

13. Katan MB, Boekschoten MV, Connor WE et al. (2009) Which are the greatest recent discoveries and the greatest future challenges in nutrition? Eur J Clin Nutr 63, 2-10.

14. Becker W, Nordhamn K, Berge S et al. (2001) Food Consumption in the Nordic Countries 1965-1998. National, Annual Food Balance Sheet Data (In Swedish with English summary). Tema Nord 2001: 527. Copenhagen: Nordic Council of Ministers.

15. Laatikainen T, Pietinen P, Valsta L et al. (2006) Sodium in the Finnish diet: 20-year trends in urinary sodium excretion among the adult population. Eur J Clin Nutr 60, 965-970.

16. Pietinen P, Valsta LM, Hirvonen T et al. (2008) Labelling the salt content in foods: a useful tool in reducing sodium intake in Finland. Public Health Nutr 11, 335-340. 